\title{
Entopeduncular Nucleus Projections to the Lateral Habenula Contribute to Cocaine Avoidance
}

\author{
Hao Li, ${ }^{1,2}$ Maya Eid, ${ }^{1}$ Dominika Pullmann, ${ }^{1}$ Ying S. Chao, ${ }^{1}$ Alen A. Thomas, ${ }^{1}$ and ${ }^{\circledR}$ Thomas C. Jhou ${ }^{1}$ \\ ${ }^{1}$ Department of Neuroscience, Medical University of South Carolina, Charleston, SC, 29425, and ${ }^{2}$ Salk Institute for Biological Studies, La Jolla, CA 92037
}

The aversive properties associated with drugs of abuse influence both the development of addiction and relapse. Cocaine produces strong aversive effects after rewarding effects wear off, accompanied by increased firing in the lateral habenula (LHb) that contributes to downstream activation of the rostromedial tegmental nucleus (RMTg). However, the sources of this LHb activation are unknown, as the $\mathrm{LHb}$ receives many excitatory inputs whose contributions to cocaine aversion remain uncharacterized. Using cFos activation and in vivo electrophysiology in male rats, we demonstrated that the rostral entopeduncular nucleus (rEPN) was the most responsive region to cocaine among LHb afferents examined and that single cocaine infusions induced biphasic responses in rEPN neurons, with inhibition during cocaine's initial rewarding phase transitioning to excitation during cocaine's delayed aversive phase. Furthermore, rEPN lesions reduced cocaine-induced cFos activation by 2 -fold in the LHb and by a smaller proportion in the RMTg, while inactivation of the rEPN or the rEPN-LHb pathway attenuated cocaine avoidance behaviors measured by an operant runway task and by conditioned place aversion (CPA). These data show an essential but not exclusive role of rEPN and its projections to the LHb in processing the aversive effects of cocaine, which could serve as a novel target for addiction vulnerability.

Key words: addiction; avoidance; cocaine; entopeduncular nucleus; lateral habenula; rostromedial tegmental nucleus

Significance Statement

Cocaine produces well-known rewarding effects but also strong aversive effects that influence addiction propensity, but whose mechanisms are poorly understood. We had previously reported that the lateral habenula $(\mathrm{LHb})$ is activated by cocaine and contributes to cocaine's aversive effects, and the current findings show that the rostral entopeduncular nucleus (rEPN) is a major contributor to this $\mathrm{LHb}$ activation and to conditioned avoidance of cocaine. These findings show a critical, though not exclusive, rEPN role in cocaine's aversive effects, and shed light on the development of addiction.

\section{Introduction}

Drugs of abuse can induce both positive and negative motivational states. In particular, many drugs show strong aversive effects after the initial rewarding properties dissipate (Koob et al., 1989; Koob and Le Moal, 2008a). For example, cocaine produces strong aversive effects roughly 15-30 min after single infusions in rodents, which manifest as robust avoidance behaviors (Ettenberg et al., 1999; Knackstedt et al., 2002; Ettenberg, 2004). These aversive effects likely have strong but complex effects on drug-seeking, helping to prevent early acquisition of drug use in some individuals, but in others contributing to escalated drug intake or

\footnotetext{
Received Mar. 26, 2020; revised 0ct. 20, 2020; accepted 0ct. 21, 2020.

Author contributions: H.L. designed research; H.L., M.E., D.P., and Y.S.C. performed research; H.L. contributed unpublished reagents/analytic tools; H.L. and A.A.T. analyzed data; H.L. and T.C.J. wrote the paper.

This work was supported by National Institutes of Health/National Institute on Drug Abuse Grants R01DA037327 and R21-DA037744 (to T.C.J.).

The authors declare no competing financial interests.

Correspondence should be addressed to Thomas C. Jhou at jhou@musc.edu.

https://doi.org/10.1523/JNEUROSCI.0708-20.2020

Copyright (C) 2021 the authors
}

relapse, because of the increased motivation to avoid the aversive "crash" postconsumption or the effects of withdrawal (Koob et al., 1989; Ettenberg et al., 1999; Knackstedt et al., 2002; Koob and Le Moal, 2008a).

An increasing number of studies have found that lateral habenula ( $\mathrm{LHb}$ ) neurons, and their targets in the rostromedial tegmental nucleus (RMTg), also called the tail of the ventral tegmental area (tVTA; Kaufling et al., 2009), are activated by negative motivational stimuli (Li et al., 2019a,b,c), including cocaine during its aversive phase (Jhou et al., 2013; Li et al., 2019a). Specifically, both LHb and RMTg neurons are activated by cocaine roughly 15-30 min after single infusions (Jhou et al., 2013; Li et al., 2019a), when cocaine is predominantly aversive. Inactivations of the LHb or RMTg greatly impair conditioned avoidance of cocaine (Jhou et al., 2013). LHb activation by cocaine may be driven partly by direct effects of cocaine on LHb neurons (Good et al., 2013; Jhou et al., 2013), but we and others have also shown that $\mathrm{LHb}$ responses to motivational stimuli are strongly driven by afferents from the rostral entopeduncular nucleus (rEPN; Shabel et al., 2012; Li et al., 2019b), with these afferents also helping drive some aversive aspects of cocaine 
withdrawal (Meye et al., 2016). In addition to the rEPN, the $\mathrm{LHb}$ also receives many excitatory inputs from other brain areas that also mediate addictive behaviors (Sheth et al., 2017; Levi et al., 2020). Thus, the relative role of the rEPN in driving $\mathrm{LHb}$ activation by cocaine, and contributing to cocaine's aversive effects, remains incompletely characterized.

In the present study, we compared cocaine-induced neural activities in several $\mathrm{LHb}$ afferent regions by examining cFos expression in combination with retrograde tracing. Using in vivo electrophysiology, we further characterized response patterns of rEPN neurons to single doses of cocaine in naive animals. Finally, we examined the effects of rEPN lesions on cocaineinduced LHb and RMTg cFos, and tested effects of rEPN and rEPN-LHb inactivation on the cocaine runway task and in cocaine conditioned place aversion (CPA). Together, our findings suggest a critical role for the $\mathrm{rEPN}$ in driving cocaine avoidance behaviors, potentially via the LHb-RMTg pathway.

\section{Materials and Methods}

Animals

All procedures were conducted under the National Institutes of Health Guide for the Care and Use of Laboratory Animals, and all protocols were approved by Medical University of South Carolina Institutional Animal Care and Use Committee. Adult male Sprague Dawley rats weighing 250-450 g from Charles River Laboratories were pair-housed in standard shoebox cages with food and water provided ad libitum until experiments started. Rats were single-housed during recording experiments and double housed for all other experiments. Rats were housed in a regular light cycle (lights on at 6 A.M., off at 6 P.M.) and tested during the light portion of this cycle. In total, 57 rats were used for these experiments, including 12 rats for cocaine cFos with LHb retrograde tracing, six for electrophysiological recordings, eight for $\mathrm{rEPN}$ lesion cFos experiments, 15 for cocaine runway experiments, and 15 animals were used in cocaine CPA designer receptor exclusively activated by designer drugs (DREADD) experiments.

\section{Surgeries}

All surgeries were conducted under aseptic conditions with under isoflurane (1-2\% at $0.5-1.0 \mathrm{l} / \mathrm{min}$ ) anesthesia. Analgesic (ketoprofen, $5 \mathrm{mg} / \mathrm{kg}$ ) was administered subcutaneously immediately after surgery. Rats were given at least $5 \mathrm{~d}$ to recover from surgery. For recording experiments, custom drivable electrode arrays were implanted above the rEPN (AP: $-2.3 \mathrm{~mm}$; ML: $2.8 \mathrm{~mm}$; DV: $-7.1 \mathrm{~mm}$ from dura). For LHb injections, $80 \mathrm{nl}$ of CTb or $400 \mathrm{nl}$ of pAAV2-hSyn-hm4D(Gi)-mcherry (AddGene) was injected into the LHb (AP: $-3.4 \mathrm{~mm}$; ML: $1.5 \mathrm{~mm}$; DV: $-4.5 \mathrm{~mm}$ from dura, $10^{\circ}$ angle). For lesion experiments, $50 \mathrm{nl}$ of $400 \mathrm{~mm}$ quinolinic acid was injected ipsilaterally into the rEPN (AP: $-2.5 \mathrm{~mm}$; ML: $3.0 \mathrm{~mm}$; DV: $-7.5 \mathrm{~mm}$ from dura). Rats were kept anesthetized with pentobarbital intraperitoneally $(55 \mathrm{mg} / \mathrm{kg}$ ) for up to $3 \mathrm{~h}$ postsurgery to reduce excitotoxic effects.

Rats were anesthetized with isoflurane and fitted with an indwelling intravenous catheter inserted into the right jugular vein, and subcutaneously passed to a guide cannula that exited via a small incision in the skin on the animal's back. After surgery, catheter patency was maintained through daily flushing with $0.05 \mathrm{ml}$ of taurolidine-citrate solution (TCS). Animals recovered for $7 \mathrm{~d}$ before behavioral testing. Catheter patency was assessed periodically through observation of the loss of the righting reflex after intravenous injection of methohexital (Brevital, $2.0 \mathrm{mg} / \mathrm{kg} / 0.1 \mathrm{ml}$ ). Rats that were unresponsive to Brevital were implanted with a new catheter using the left jugular vein.

\section{Perfusions and tissue sectioning}

Rats in all experiments were killed with an overdose of isoflurane and perfused transcardially with $10 \%$ formalin in $0.1 \mathrm{M}$ PBS, pH 7.4. In electrophysiology experiments, $100-\mu \mathrm{A}$ current was passed through the recording wires just before perfusion, facilitating visualization of electrode tips. Brains were removed from the skull, and postfix in $10 \%$ formalin for $24 \mathrm{~h}$ before equilibration in $20 \%$ sucrose solution. Brains were cut into $40-\mu \mathrm{m}$ sections on a freezing microtome, and sections were stored in phosphate buffered saline with $0.05 \%$ sodium azide.

\section{Immunohistochemistry}

Free-floating sections were immunostained for $\mathrm{CTb}$ or cFos by overnight incubation in goat anti-CTb (List Biological Laboratories, 7032A9, 1:50,000 dilution) or rabbit anti-cFos (Millipore, ABE457, 1:1000 dilution) primary in PBS with $0.25 \%$ Triton $\mathrm{X}-100$ and $0.05 \%$ sodium azide. Afterwards, tissue was washed three times in PBS and incubated in biotinylated donkey-anti-goat or anti-rabbit secondary (1:1000 dilution, Jackson ImmunoResearch) for $30 \mathrm{~min}$, followed by three 30 -s rinses in PBS, followed by $1 \mathrm{~h}$ in avidin-biotin complex (Vector). For CTb-staining, tissue was then rinsed in sodium acetate buffer $(0.1 \mathrm{M}, \mathrm{pH} 7.4)$, followed by incubation for $5 \mathrm{~min}$ in $1 \%$ diaminobenzidine (DAB). For cFos, nickel and hydrogen peroxide (Vector) were added to reveal a blue-black reaction product.

\section{Behavioral training for electrophysiological recordings}

Rats were food restricted to $85 \%$ of their ad libitum body weight and trained to associate distinct auditory cues with either a sucrose pellet or no outcome. Behavior was conducted in standard Med Associates chambers. Reward and neutral cues were a $1-\mathrm{kHz}$ tone $(75 \mathrm{~dB})$ and white noise $(75 \mathrm{~dB})$, respectively. Reward and neutral cues were presented for $2 \mathrm{~s}$ each, followed immediately by a sucrose pellet ( $45 \mathrm{mg}$, BioServ) or nothing, respectively. The two trial types were randomly intermixed with 30 -s intervals between successive trials. A "correct" response was scored if animals nosepoked into the food tray within $2 \mathrm{~s}$ after reward cue offset, or withheld a response for $2 \mathrm{~s}$ after neutral cue onset. Rats were trained with 100 trials per session, one session per day, until they achieved $80 \%$ accuracy. Then, rats underwent surgeries to implant recording electrodes. After recovery, rats were then trained with one extra session in which neutral cue trials were replaced by aversive trials consisting of a 2 -s $8-\mathrm{kHz}$ tone $(75 \mathrm{~dB})$ followed immediately by a $10-\mathrm{ms} 0.6$ $\mathrm{mA}$ footshock.

\section{Electrophysiological recordings}

After final training, electrodes consisted of a bundle of sixteen $18-\mu \mathrm{m}$ Formvar-insulated nichrome wires (A-M Systems) attached to a custom-machined circuit board. An additional 37-gauge ground wire was attached to a gold-plated pin (Newark Electronics), which was implanted into the cortex. Recordings were performed during once-daily sessions, and electrodes were advanced $160 \mu \mathrm{m}$ (half-turn of the screw) at the end of each session, allowing us to record from multiple sites along a vertical track in each animal. The recording apparatus consisted of a unity gain headstage (Neurosys LLC) whose output was fed to preamplifiers with high-pass and low-pass filter cutoffs of $300 \mathrm{~Hz}$ and $6 \mathrm{kHz}$, respectively. Analog signals were converted to 18 -bit values at a frequency of $15.625 \mathrm{kHz}$ using a PCI card (National Instruments) controlled by customized PC-based acquisition software (Neurosys LLC). Spikes were initially detected via thresholding to remove signals less than 2 -fold above background noise levels, and signals were further processed using principal component analysis performed by NeuroSorter software, allowing manual "clustering" of spikes into putative neural "units." Units were accepted only if they had a refractory period, determined by $<0.2 \%$ of spikes occurring within $1 \mathrm{~ms}$ of a previous spike, as well as by the presence of a large central notch in that unit's auto-correlogram. Units with significant drifts in firing rates or spike amplitudes during a single recording session were excluded, along with units that showed $10 \%$ or greater overlap with other units in cross-correlogram analyses. Since the shock duration used in the present study was $10 \mathrm{~ms}$, the first $10 \mathrm{~ms}$ of data after footshock were excluded from analysis, because of contamination by shock artifacts.

For the discrimination paradigm, once rats achieved at least $80 \%$ accuracy in reward trials, they were trained to respond to an $8-\mathrm{kHz}$ tone $(75 \mathrm{~dB})$ lasting for $2 \mathrm{~s}$ followed by a mild footshock $(0.6 \mathrm{~mA})$. During testing, rats were again placed on mild food deprivation, and recordings obtained in sessions consisting of a mixture of 150 reward trials, neutral trials, and shock trials randomly selected. One $0.75-\mathrm{mg} / \mathrm{kg}$ cocaine 
infusion intravenously was given to rats at least $30 \mathrm{~min}$ after the cued discrimination task, with recordings continuing for $45 \mathrm{~min}$ after infusion.

We recorded from six rats in total with each rat undergoing up to four recording sessions. After histologic verification of recording sites in the EPN, a total of 10 recording sessions from 6 rats were included for subsequent analysis. On average, we recorded three neurons per session, yielding a range of two to eight usable neurons per animal. Neural data acquired in all sessions and all animals were analyzed as independent neurons, even if they came from the same animal.

Cocaine-induced cFos induction and quantification

Animals used for cFos experiments were habituated to handling for $3 \mathrm{~d}$, then given one $0.75 \mathrm{mg} / \mathrm{kg}$ intravenous cocaine infusion and returned to its home cage, then perfused $1 \mathrm{~h}$ after.

Upon the competion of cFos immunostaining, two slices of brain tissues which contain the center of each region were selected for cell counting based on a standard rat atlas (Paxinos and Watson, 1998). The numbers of cFos-positive neurons and $\mathrm{CTb}$-positive neurons for each region were independently counted by two human observers.

\section{$C P A$}

This task used a three-chambered place preference apparatus as previously described (Jhou et al., 2009). On day 1 , animals were placed in the small central chamber, and allowed to explore the apparatus freely for $15 \mathrm{~min}$. Subsequently, doors between chambers were closed, and one (non-central) chamber was designed to be "paired" with cocaine infusions, and the other "unpaired" chamber associated with saline infusions. Chamber-drug pairings were counterbalanced between animals. Animals were then placed into each chamber for a total of eight conditioning sessions (two per day), alternating between cocaine and saline in counterbalanced order (i.e., half of animals received cocaine first, the other half saline first). Rats received an infusion of intravenous cocaine $(0.75 \mathrm{mg} / \mathrm{kg})$ or saline and remained in their home cage for $15 \mathrm{~min}$ before being placed into the paired or unpaired side of the chamber, and then were allowed to remain in chambers for another $15 \mathrm{~min}$. Half of the rats received cocaine infusion for the first session of the day, while the rest of the half received saline infusion. In the final session (i.e., test day: day 5), animals were placed into the central compartment of the apparatus and allowed to freely explore all three chambers for $15 \mathrm{~min}$. Time spent in each compartment was recorded.

\section{Runway operant cocaine-seeking}

Methods are similar to our previous work (Jhou et al., 2013). Briefly, the runway consists of two opaque plastic compartments ("start" and "goal") connected by a $170-\mathrm{cm}$-long corridor. Rats are tethered to an intravenous line, placed into the start box, after which doors are opened, allowing free exploration of the apparatus. Entry into the goal box causes doors to close and a syringe pump to deliver cocaine $(0.75 \mathrm{mg} / \mathrm{kg}$, i.v.; same dose as in CPA). Rats remained in the goal compartment for another $5 \mathrm{~min}$ before returning to their home cage. Failure to enter the goal compartment after 15 min resulted in a timeout. Rats received up to two trials per day with at least $4 \mathrm{~h}$ apart, ensuring that animals ran in a cocaine-free state. Before cocaine testing, animals were habituated to the runway for three sessions or until they reached the goal box within $60 \mathrm{~s}$.

\section{Microinfusion}

A 26-gauge stainless steel internal cannula (PlasticsOne) projecting $1 \mathrm{~mm}$ beyond the tip of the guide cannula was connected to a syringe pump (Harvard Apparatus). Clozapine N-oxide (CNO; 1 mm, NIDA Drug Supply Program) was diluted in saline solution containing 2\% dimethylsulfoxide (DMSO). CNO was infused slowly into both sides of the LHb (500 $\mathrm{nl}$ per side) over a duration of 2 min beginning $10 \mathrm{~min}$ after cocaine infusions and $5 \mathrm{~min}$ before rats were placed into the conditioning chamber. CNO infusions were omitted for the saline conditioning sessions to minimize the tissue damages from microinjections. For muscimol infusions, muscimol $(0.05 \mathrm{nmol}$ in $500 \mu \mathrm{l}$, Sigma) or PBS (equal volume) infusions were infused bilaterally over $2 \mathrm{~min}$ in the home cage at $10 \mathrm{~min}$ after cocaine delivery, to overlap with the aversive phase of cocaine.
Rats were excluded if cannulae placements missed on either (or both) sides.

\section{Statistical analysis of electrophysiological and behavioral data}

Neurons with large drifting of the microwire electrodes during recordings were excluded from further analysis. Electrophysiology data were first tested for normality, then transformed into ranked forms if data failed tests of normality ( $p<0.05$, D'Agostino-Pearson test). Significant responses in neural firing were determined by a threshold of $p<0.05$ for each neuron's firing rate versus baseline (Wilcoxon signed-rank). Data were tested for normality ( $p>0.05$, D'Agostino-Pearson test), and were analyzed using parametric tests. One-way or two-way ANOVA with Holm-Sidak correction for multiple comparisons, and two-tailed $t$ test were used to compare across experimental conditions, respectively, if not otherwise specified. Calculations were performed using MATLAB (MathWorks) and Prism 7 software (GraphPad).

\section{Results}

\section{LHb afferents are activated by acute cocaine infusion}

In order to examine which LHb afferents might drive its responses to cocaine, we first examined the induction of the immediate early gene cFos, a proxy for neural activation, in several $\mathrm{LHb}$ afferents in response to a single cocaine infusion $(0.75 \mathrm{mg} /$ kg, i.v.; Fig. 1A). We injected the retrograde tracer cholera toxin subunit $\mathrm{B}(\mathrm{CTb})$ into the $\mathrm{LHb}$ and tabulated $\mathrm{CTb}$-and cFos immunoreactive neurons in several LHb afferents, particularly the rEPN, ventral pallidum (VP), lateral preoptic area (LPO), and lateral hypothalamus $(\mathrm{LH})$. We found robust afferents to the $\mathrm{LHb}$ from all four sources, with the $\mathrm{LH}$ expressing the highest proportion of $\mathrm{CTb}$ neurons but with substantial numbers in the other three as well ( $\mathrm{LH}$ vs $\mathrm{rEPN}: p=0.01, t_{(33)}=3.97$; $\mathrm{LH}$ vs LPO: $p=0.01, t_{(33)}=4.11 ; \mathrm{LH}$ vs VP: $p=0.072, t_{(33)}=2.75 ; \mathrm{rEPN}$ vs LPO: $p=0.84, t_{(33)}=0.33$; rEPN vs VP: $p=0.73, t_{(33)}=0.98$; LPO vs VP: $p=0.84, t_{(33)}=0.54$, repeated measurement one-way ANOVA with Holm-Sidak multiple comparison test, $F_{(3,33)}=$ $7.699, p=0.0005$; Fig. $1 B)$. Compared with the saline group $(n=6)$, we found that cocaine infusions $(n=6)$ significantly increased proportions of cFos-expressing neurons in CTb-labeled neurons in the rEPN $(73.3 \pm 4.6 \%$ vs $12.3 \pm 1.2 \%)$ and the VP $(32.7 \pm 3.3 \%$ vs $12.7 \pm 1.1 \%)$, but not LH or LPO $\left(p<0.0001, t_{(40)}=-5.623\right.$ for the VP; $p<0.0001, t_{(40)}=-17.15$ for the $\mathrm{rEPN} ; p=0.88, t_{(40)}=$ -0.14 for the LPO; $p=0.58, t_{(40)}=0.937$ for the LH; two wayANOVA with Holm-Sidak multiple comparison test, interaction: $F_{(3,30)}=58.8, p<0.0001$; region: $F_{(3,30)}=55.54, p<0.0001$; treatment: $F_{(1,10)}=242.2, p<0.0001$; Fig. $\left.1 C, D\right)$.

\section{rEPN activation occurs during cocaine's aversive phase}

Although we identified the rEPN as having a large proportion of neurons activated by cocaine, the poor temporal resolution of cFos made it uncertain whether this activation occurred during cocaine's rewarding phase and/or its aversive phase (or neither). Hence, we used electrophysiological recordings in awake behaving rats to further characterize rEPN responses to cocaine (Fig. $2 A, B)$. Driveable recording wires were implanted into the EPN, as subsequently delineated by the presence of $\mathrm{CTb}$ and the absence of parvalbumin immunostaining (Rajakumar et al., 1994). Animals were first trained to respond to two distinct auditory tones predicting either a food pellet or nothing (reward trials vs neutral trials). After $8 \mathrm{~d}$ of training, almost all animals responded correctly on $>80 \%$ of trials, defined as nosepoking into the reward receptacle within $2 \mathrm{~s}$ after reward cue onset, or withholding poking during the same period after neutral cue onset. Animals were then trained with a third auditory tone predicting a very brief mild footshock (10-ms duration, $0.6 \mathrm{~mA}$; Fig. 2C,D). 
A

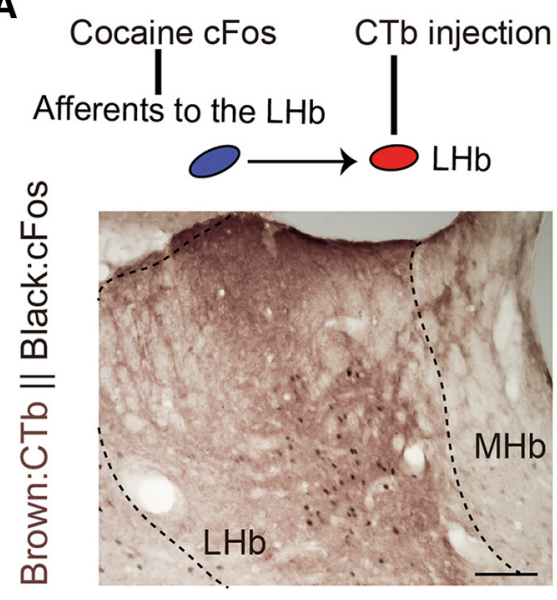

B

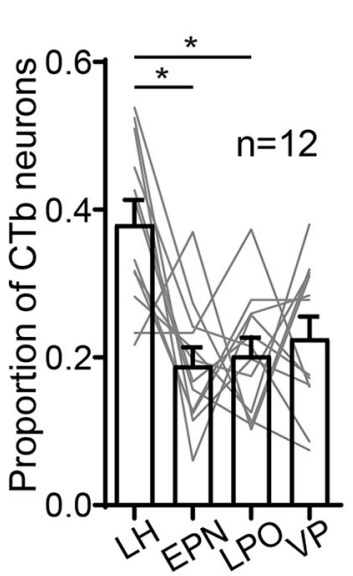

C

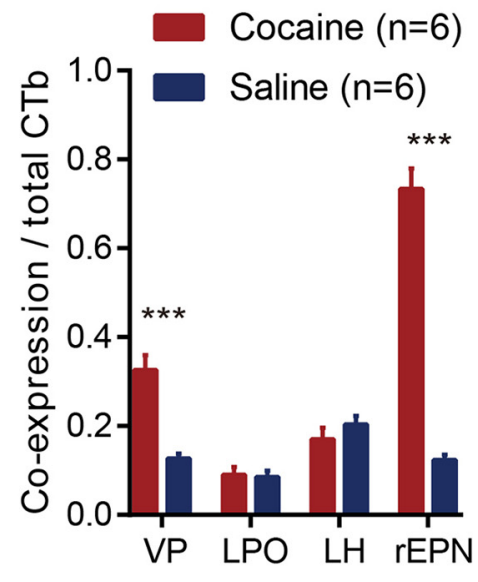

D
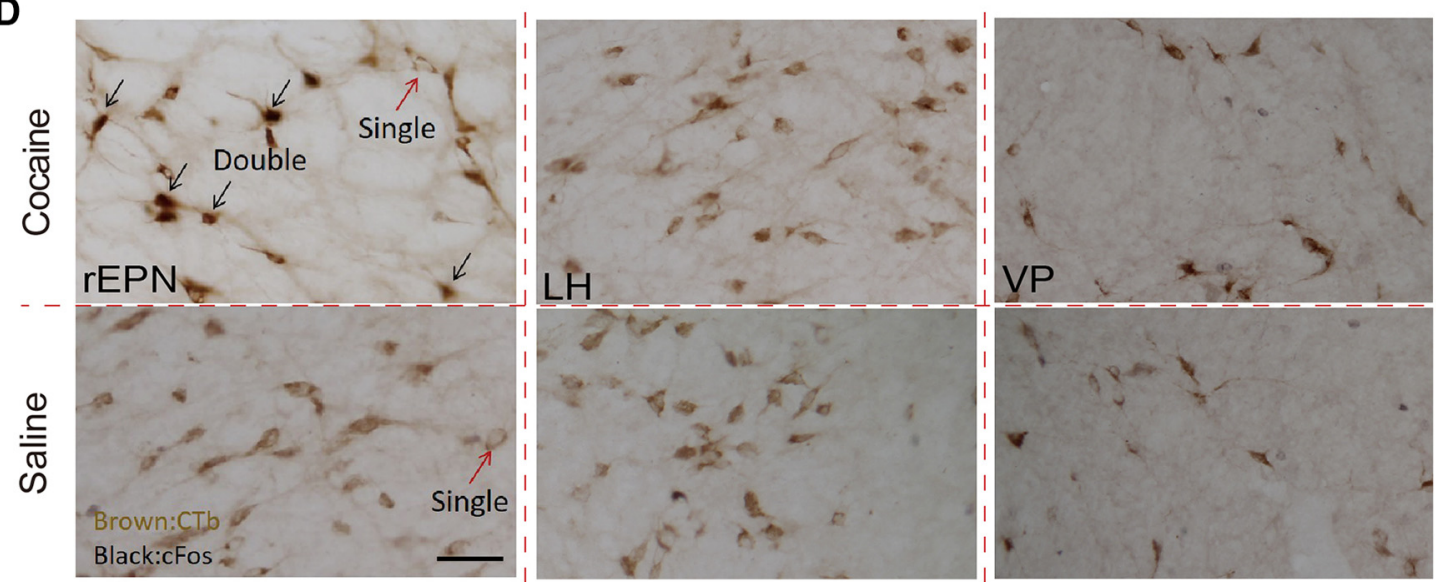

Figure 1. LHb afferents are activated by acute cocaine infusion. $A$, Schematics of experimental procedure and cocaine cFos induction, and representative photomicrograph of immunostaining of $\mathrm{CTb}$ injecton site in the LHb. B. The LH showed the highest proportion of CTb-labeled neurons among these LHb afferent. C, Proportions of CTb-labeled neurons expressing CFos in the VP, LPO, LH, and rEPN, showing the largest cocaine-induced increases in LHb-projecting neurons in the rEPN. D, Representative photomicrographs of immunostaning of CTb and CFos in the rEPN, LH, and VP. Black arrows indicate neurons immunoreactive for both CTb and CFos, red arrows indicate single labeled CTb neurons. Scale bars: $100 \mu \mathrm{m}(\boldsymbol{A}, \boldsymbol{D})$.

rEPN neurons were recorded in response to 150 total trials (reward, neutral, and shock trials) as well as a subsequent cocaine session in which animals received either a single intravenous cocaine or saline infusion after a 30-min baseline. Consistent with rEPN encoding of negative motivational valence (Li et al., 2019b), we observed that rEPN neurons exhibited monotonic encoding of cue valence. Specifically, population averages of rEPN neurons showed the greatest firing after shock-predictive cues, lowest firing after reward-predictive cues, and intermediate firing rates after neutral cues $\left(p=0.0105, t_{(54)}=2.649\right.$ for reward cue vs neutral cue; and $p=0.0132, t_{(54)}=2.561$ for neutral cue vs shock cue, repeated measurement one-way ANOVA with Holm-Sidak multiple comparison test, $F_{(2,54)}=9.548, p=0.0003$; Fig. $\left.2 E\right)$. When rEPN neurons were analyzed individually, the most common response was an inhibition to reward-predictive cues (13/28 neurons), while excitation or non-response was less common (5/28 and 10/28 neurons, respectively).

Notably, responses to reward, neutral, and shock cues were analyzed in different time windows $(0-100 \mathrm{~ms}$ for neutral and shock cues, and $200-400 \mathrm{~ms}$ for reward cues) consistent with prior findings that the EPN, and its downstream targets in LHb and RMTg, respond more slowly to reward-related cues than other cues (Jhou et al., 2009; Bromberg-Martin et al., 2010; Li et al., 2019a,b,c). Hence, our tabulation of rEPN responses to reward cues does not include the earliest portion of that response, which sometimes includes a small transient excitation. Notably, this transient excitation is much smaller than that seen after shock cues and does not distinguish between reward and neutral cues (Fig. $2 E$, red vs gray traces; $p=0.56, t_{(54)}=0.583$ for reward cue vs neutral cue; $p=0.003, t_{(54)}=3.017$ for reward cue vs shock cue; $p=0.018, t_{(54)}=2.434$ for shock cue vs neutral cue; repeated measurement one-way ANOVA with Holm-Sidak multiple comparison test, $\left.F_{(2,54)}=5.122, p=0.0098\right)$, more consistent with cue generalization than valence encoding per se.

We next examined $\mathrm{rEPN}$ responses to cocaine infusions. We found that the majority of rEPN neurons (14/20) were inhibited during the first $10 \mathrm{~min}$ after cocaine infusion compared with baseline $(z$ score $=-2.05, p=0.04$ and $-3.56, p=0.0003$ for biphasic and inhibited groups). A much different pattern emerged 1530 min postinfusion, when 10 of the 14 neurons that were inhibited during the first $10 \mathrm{~min}$ showed significant excitation compared with baseline firing $(z$ score $=2.913, p=0.003)$, while the remaining four slowly returned to baseline. Notably the six neurons that did not respond during the first $10 \mathrm{~min}$ remained unresponsive $(z$ score $=0.488, p=0.625)$ Notably, 10 neurons showed both an inhibition during the first $0-10 \mathrm{~min}$ and excitation during $15-30 \mathrm{~min}$ 
A

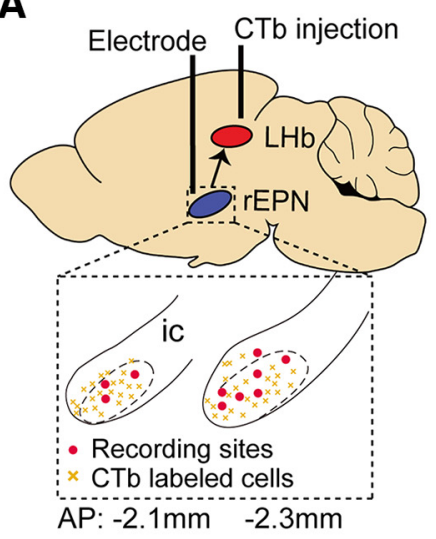

B

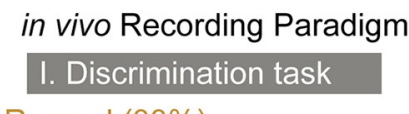

Reward (33\%)

Tone A Food Pellet

Shock (33\%)

Tone B 10ms Shock

Neutral $(33 \%)$
Tone C Nothing
$2 \mathrm{~s}$

II. Cocaine infusion

i.v. infusion of cocaine or saline 30 minutes after the last discrimination trial
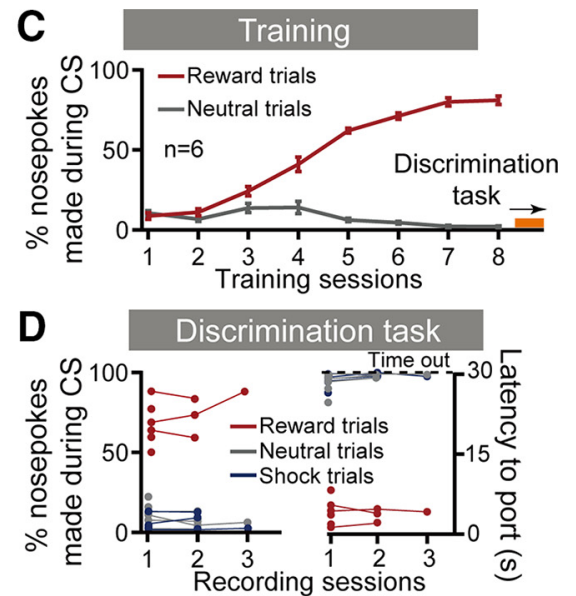

Cocaine infusion

\section{E Discrimination task}

$\mathbf{F}$
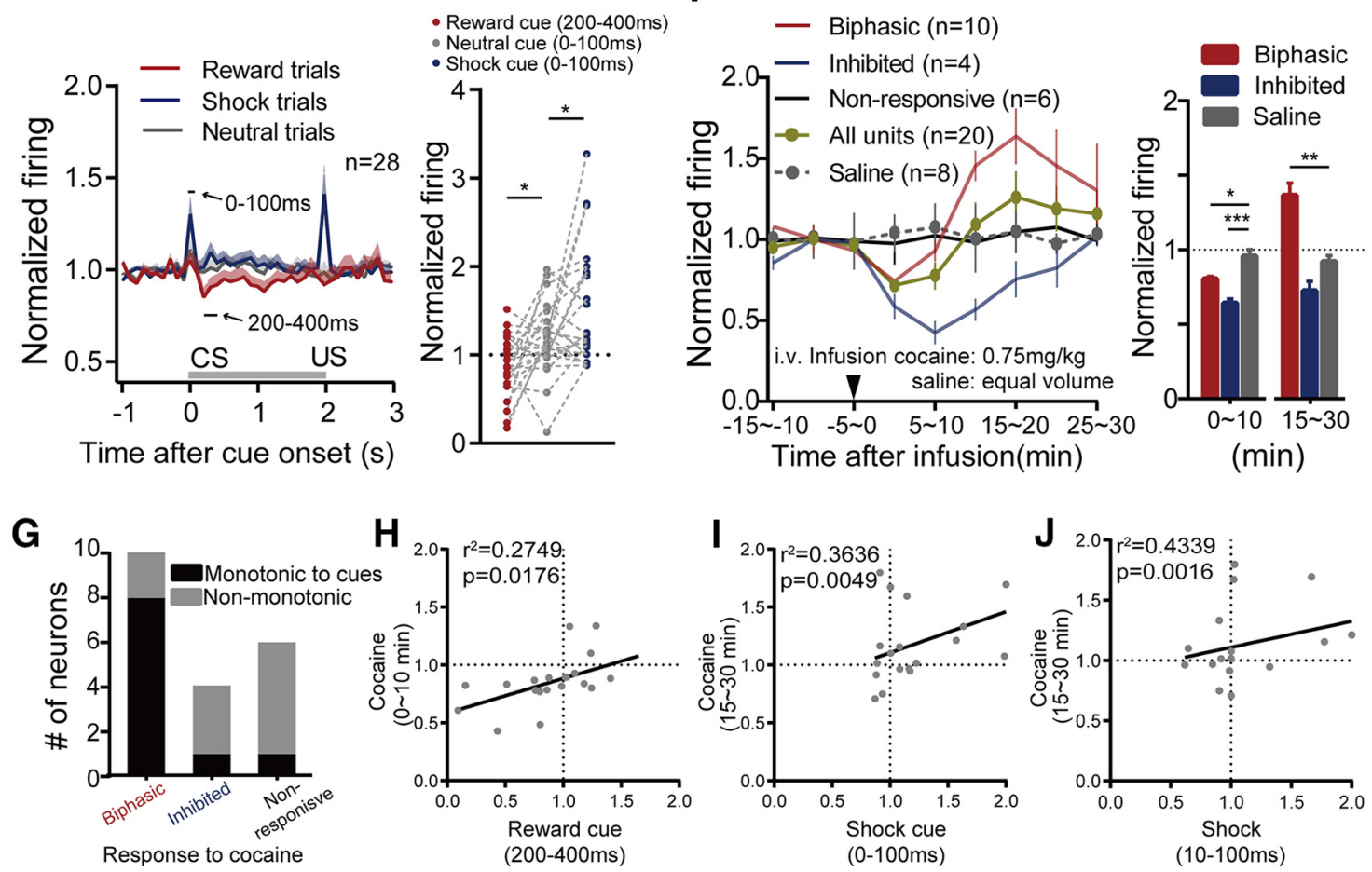

Figure 2. rEPN neuron activation during cocaine's delayed aversive phase. A, Schematic of recording experiments in rEPN (upper panel) and recording sites in rEPN (lower panel), showing that recording sites were located in the LHb-projecting region. B, Schematic of recording paradigm. Animals performed a discrimination task containing of a block of 100 reward (food), neutral, and shock trials, followed by a single dose of cocaine or saline intravenous infusion. $C$, Behavioral outcomes during reward training. Animals were first trained with reward and neutral trials for $8 \mathrm{~d}$ before adding the shock trials for discrimination task. Trials were successful if animals entered food port within $2 \mathrm{~s}$ after reward cue onset, or withheld entry for $2 \mathrm{~s}$ after neutral or shock cues. $\boldsymbol{D}$, Behavioral outcomes for individual rats during each recording session of discrimination task. Each dot in reward, neutral, and shock trials represents one individual rat, and lines connect sessions from the same rats. $E$, Average responses of rEPN neurons to reward, neutral, and shock trials in the discrimination task. Black bars indicate the analysis windows used in the adjacent panel. Reward cue responses were averaged in a 200- to 400-ms time window after cue onset, while neutral and shock cue response were averaged during a 0 - to 100 -ms time window. $\boldsymbol{F}$, Among these neurons, 10/20 showed biphasic responses to cocaine with inhibition 0-10 min and excitation 15-30 min after injections (red trace), while 4/20 showed only inhibition (blue trace) and 6/20 showed excitation or no response to cocaine (black trace). These neurons did not respond to saline infusion (dashed trace). Responses of biphasic and inhibited neurons $0-10$ min postcocaine were significantly decreased compared with saline, and response of biphasic neurons $15-30$ min postcocaine were significantly increased. G, A majority (8 out of 10) of biphasic

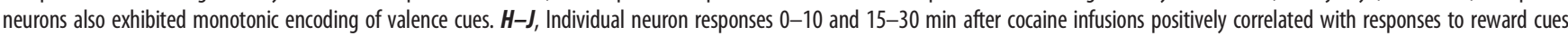
and shock cues/shocks during 200- to 400- and 0- to 100-ms poststimulus time windows.

postinfusion, reflecting a biphasic response (Fig. $2 F$ ). Out of these 10 neurons, eight of them also exhibited the monotonic response to valence-predictive cues (Fig. $2 G$ ). The time course of rEPN firing paralleled previous recordings in the LHb and RMTg, where neurons were also initially inhibited and subsequently activated by cocaine infusions, corresponding to the time course of cocaine's motivational switch from rewarding to aversive (Jhou et al., 2013; Li et al., 2019a). Furthermore, individual rEPN neural responses to reward-predictive cues positively correlated with their averaged responses $0-10 \mathrm{~min}$ after cocaine infusion (rewarding phase; $r^{2}=$ $0.2749 ; p=0.0176$ ), while their responses to shock-predictive cues or footshocks positively correlated with responses $15-30 \mathrm{~min}$ after cocaine infusion (aversive phase; $r^{2}=0.3636 ; p=0.0049$ for shock cues, and $r^{2}=0.4339 ; p=0.0016$ for footshocks), further suggesting that $\mathrm{rEPN}$ neuron biphasic responses to cocaine reflect cocaine's biphasic motivational valence (Fig. $2 H-J$ ). Notably, in all analyses of postshock firing, we omitted the first 10 -ms time bin from analysis, because of the presence of electrical artifacts from the shock itself. 
A
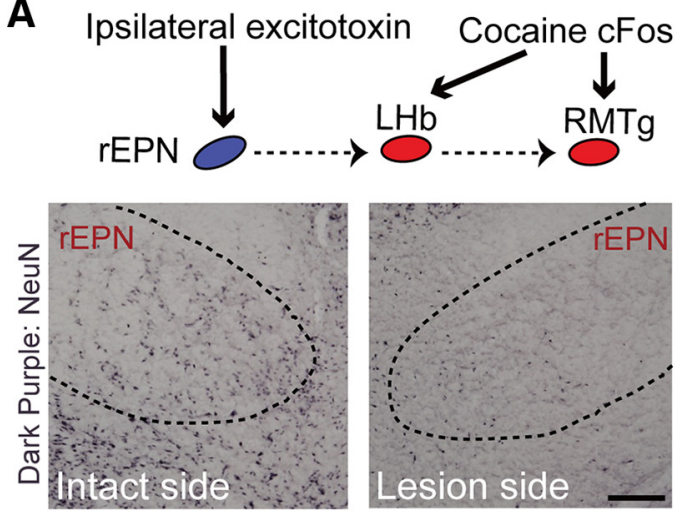

\section{Intact side}

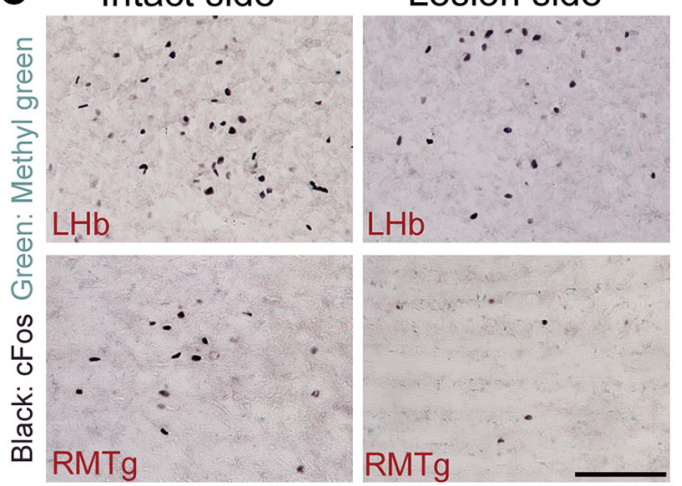

B

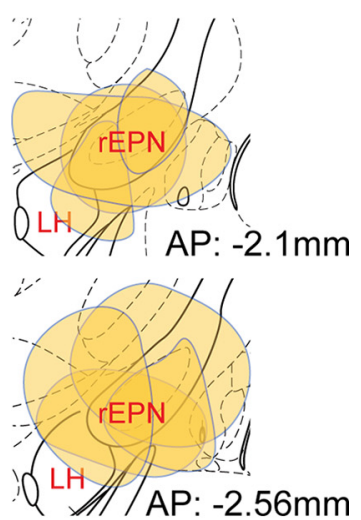

causing the delivery of a single infusion $(0.75 \mathrm{mg} / \mathrm{kg})$ of cocaine (Fig. $4 A)$. The latency to reach the goal box was recorded, along with the number of run direction reversals, as a proxy of the motivation to seek cocaine. We tested two groups of animals with bilateral infusions of either muscimol $(n=6)$ or vehicle $(n=9)$ into the rEPN, given $10 \mathrm{~min}$ after animals arrived in the goal box and received cocaine, thus overlapping with cocaine's aversive phase (Fig. 4B). Vehicle-treated animals' run latencies started to increase by the second or third trial, consistent with the development of conditioned avoidance responses (Ettenberg et al., 1999). In contrast, muscimol-treated animals remained significantly faster $(<60 \mathrm{~s})$ than the vehicle group for the first 7 trials $\left(p=0.036, t_{(17)}=2.618\right.$ for muscimol vs vehicle; $p=0.653, t_{(17)}=0.4582$ for missed hits vs vehicles; one-way ANOVA with Holm-Sidak multiple comparison test, $\left.F_{(2,17)}=3.946, p=0.04\right)$. Starting trial 8, muscimol infusions were replaced by vehicle infusions, and animals' latencies rapidly increased, eventually becoming similar to those of control animals $\left(p=0.811, t_{(17)}=\right.$ 0.586 for muscimol vs vehicle; $p=0.841$, $t_{(17)}=0.2039$ for missed hits vs vehicles; one-way ANOVA with Holm-Sidak multiple comparison test, $F_{(2,17)}=0.343$, $p=0.7147$; Fig. $4 C$ ), suggesting that the reduced latencies were not because of global deficits in avoidance learning. Notably, all rEPN microinfusions (saline

\section{rEPN lesion diminished cocaine-induced cFos in $\mathrm{LHb}$ and} RMTg neurons

Next, we examined whether the rEPN is necessary for driving $\mathrm{LHb}$ and RMTg activation by cocaine. We unilaterally lesioned the rEPN with an excitotoxin (quinolinic acid) and measured the ratio of ipsilateral to contralateral cFos expression in the $\mathrm{LHb}$ and RMTg induced by the same dose of cocaine. A control group received the same volume of PBS injected into the rEPN ( $n=4$ for both lesion and control groups; Fig. $3 A, B$ ). Lesions reduced $\mathrm{LHb}$ cFos by more than 2 -fold compared with the intact hemisphere, while RMTg cFos was also significantly reduced, but by a much smaller proportion $\left(p<0.0001 ; t_{(4)}=6.729\right.$ and $p=0.003 ; t_{(4)}=3.685$ for the LHb and the RMTg, respectively, two-way ANOVA with Holm-Sidak multiple comparison test, interaction: $F_{(1,12)}=4.631, p=0.052$; region: $F_{(1,12)}=1.965$, $p=0.1863$; treatment: $\left.F_{(1,12)}=54.23, p<0.0001\right)$. In contrast, control rats showed equal amounts of cFos on both sides (Fig. 3C,D).

\section{The rEPN and its projections to the LHb mediate cocaine avoidance behaviors}

Prior electrophysiological and lesion studies strongly suggest that the rEPN plays an important role in driving cocaine avoidance behaviors. Hence, we directly tested the rEPN role in cocaine avoidance using an operant runway task, which is highly sensitive to the aversive effects of cocaine (Ettenberg, 2009; Jhou et al., 2013). In this task, animals were placed into a chamber at one end of a five-foot corridor, and allowed to run to a goal chamber at the opposite end, whereupon a photobeam is interrupted or muscimol) were delivered after reaching the goal box; hence, any influences on running speed would not be because of acute motoric effects of microinfusions, but instead were because of influences on conditioning that occurred in previous trials. Nonetheless, we also measured run reversals and average run speed to control for possible effects on motor performance. We found that rEPN inactivation significantly reduced the number of run reversals during the first seven cocaine sessions $(p=0.0003$, $t_{(17)}=4.844$ for muscimol vs vehicle; $p=0.147, t_{(17)}=1.518$ for missed hits vs vehicles; one-way ANOVA with Holm-Sidak multiple comparison test, $F_{(2,17)}=11.79, p=0.0006$; Fig. $\left.4 D\right)$, while the relative speed in each session (calculated as the total distance traveled divided by latency), was not different between the two groups $\left(p=0.613, t_{(17)}=0.5146\right.$ for muscimol vs vehicle; $p=0.4622, t_{(17)}=1.151$ for missed hits vs vehicles; one-way ANOVA with Holm-Sidak multiple comparison test, $F_{(2,17)}=$ $0.6624, p=0.5292$; Fig. $4 E$ ).

We further evaluated whether the abolishment of the avoidance to cocaine in the runway was because of the reduction of the aversive effects of cocaine via rEPN projections to the $\mathrm{LHb}$ by using pathway-specific inactivation of LHb with DREADDs in a CPA paradigm. Previous studies showed that cocaine causes a strong CPA starting $15 \mathrm{~min}$ after infusion (Ettenberg et al., 1999). We therefore bilaterally injected inhibitory DREADDs (hM4Di) into the EPN and inhibited the rEPN-LHb pathway by microinfusion of CNO into the LHb. Animals injected with hM4Di $(n=8)$ or mCherry $(n=7)$ were conditioned with cocaine 
and saline in opposite sides of the chamber $15 \mathrm{~min}$ after infusion for eight continuous days, and $\mathrm{CNO}$ was infused $5 \mathrm{~min}$ before conditioning to the cocaine paired chamber (Fig. 4F-I). We found that animals in the control group developed CPA to cocaine, while inactivating EPN terminals in the LHb produced a significant shift from conditioned aversion to conditioned place preference measured by both time spent in the cocaine paired chamber and numbers of entries to the chamber (for time spent: $p<0.0001, t_{(15)}=7.493$ for $\mathrm{CNO}$ vs controls; $p=0.273, t_{(15)}=$ 1.141 for missed hits vs controls; for the number of entries: $p=0.016, t_{(15)}=3.02$ for $\mathrm{CNO}$ vs controls; $p=0.84, t_{(15)}=$ 0.2051 for missed hits vs controls; oneway ANOVA with Holm-Sidak multiple comparison test, $F_{(2,15)}=30.87, p<$ 0.0001 and $F_{(2,15)}=5.159, p=0.0197$, respectively; Fig. 4J,K).

\section{Discussion}

The present results show that single infusions of cocaine-induced robust cFos expression in LHb-projecting rEPN neurons, and to a lesser extent VP, but not other $\mathrm{LHb}$ afferents. Using in vivo recordings, we further found that cocaine produced biphasic inhibitory/excitatory responses in a subset of rEPN neurons whose time course paralleled cocaine's biphasic rewarding and aversive motivational effects. Excitotoxic rEPN lesions markedly reduced cocaine-induced cFos in the $\mathrm{LHb}$, with a more modest reduction in RMTg, and inactivation of the rEPN impaired acquisition of conditioned avoidance to cocaine in a runway operant task. Furthermore, using pathwayspecific DREADDs, we showed that the rEPN mediates cocaine avoidance via its projections to $\mathrm{LHb}$. These results strongly indicate the important role of the rEPN in mediating cocaine avoidance behavior.

The major rEPN projection to LHb has been extensively studied in mood disorders. In particular, rEPN terminals corelease glutamate and GABA into the $\mathrm{LHb}$, with the glutamatergic component becoming increasingly predominant in rodent models of depression (Li et al., 2011; Shabel et al., 2012). Such shifts between glutamatergic and GABAergic transmission have also been observed in chronic cocaine withdrawal and relapse (Meye et al., 2016), making the rEPN a potential candidate to drive LHb activity during cocaine's aversive phase. Indeed, we found that among major LHb afferents, the rEPN had the highest cocaine-induced cFos protein expression. In vivo electrophysiological recordings further

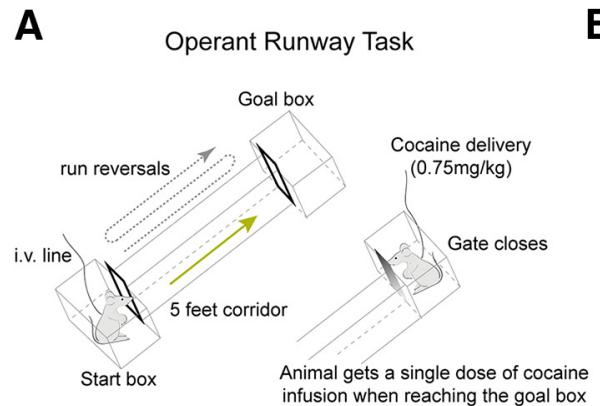

B rEPN inhibition procedure
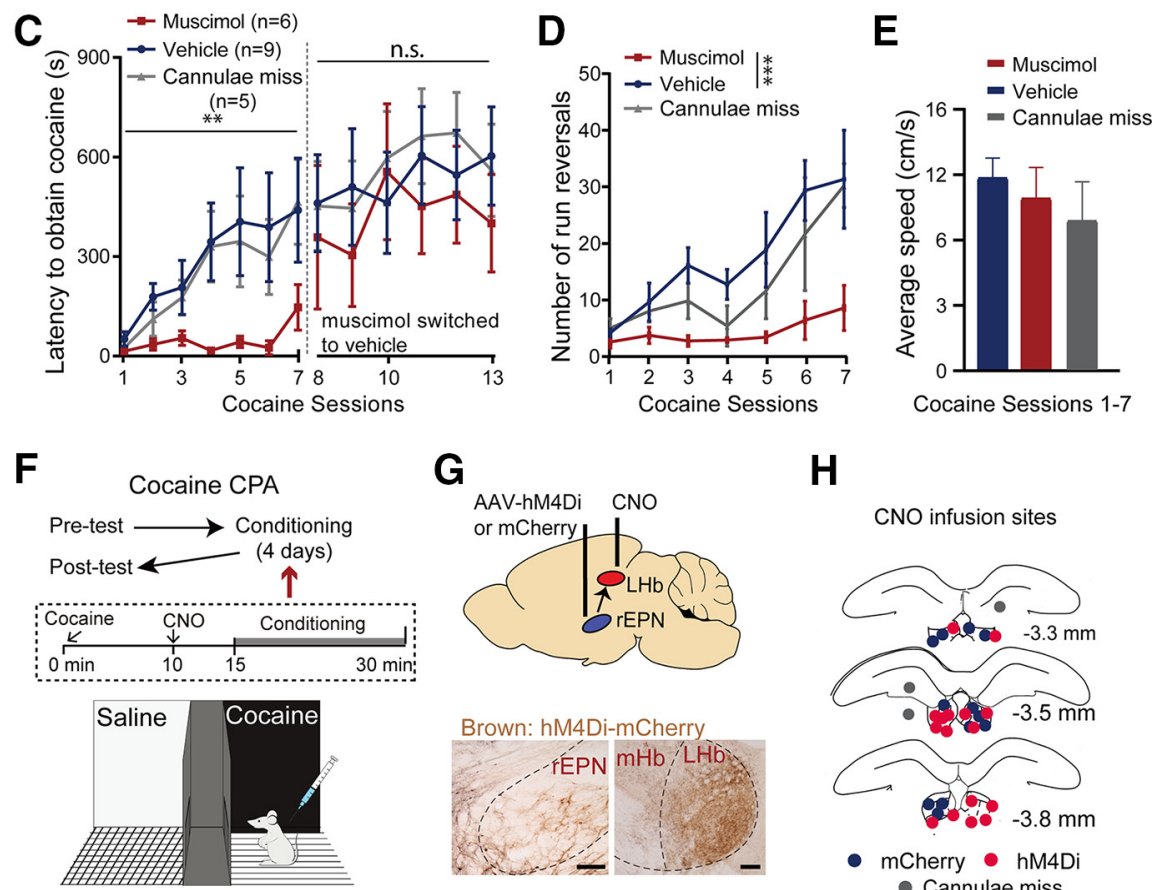

H
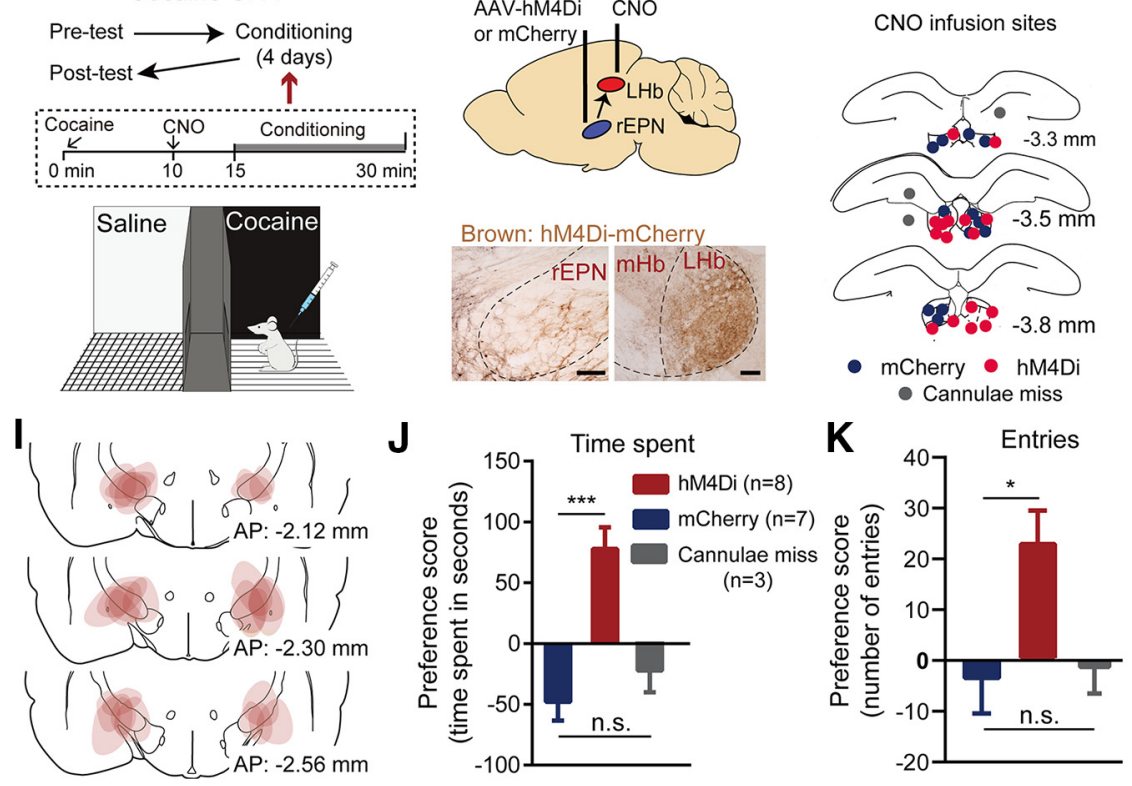

Figure 4. The rEPN and its projections to the LHb mediate cocaine avoidance behaviors. $A$, Cocaine runway paradigm. In each session, animals started at the start box and received a single cocaine infusion uponreaching the goal box, or timed out after $900 \mathrm{~s}$ and was manually placed into goal box and given cocaine. B, Muscimol (red dots) and vehicle (blue dots) infusion sites in the rEPN. rEPN muscimol infusions blocked development of cocaine avoidance $(\boldsymbol{C})$ and reduced run direction reversals (D) during sessions 1-7. E, Locomotion averaged over sessions 1-7 was not different between groups. $\boldsymbol{F}$, Behavioral paradigm of CPA. Animals received CNO infusion into the LHb $10 \mathrm{~min}$ after cocaine infusion and were placed into paired chamber 5 min after CNO infusion for 15 min. $\boldsymbol{G}$, Photomicrographs of immunostaining of mCherry in the rEPN and LHb. $\boldsymbol{H}$, CNO (red dots) and vehicle (blue dots) infusion sites in the LHb. I, Illustration of the extent of the virus expression for eight hM4Di animals. $\boldsymbol{J}, \boldsymbol{K}$, Inactivation of rEPN-LHb pathway blocked CPA induced by acute cocaine withdrawal. Preference sores are calculated by the difference in time spent and number of entries to the cocaine-paired chamber before and after the conditioning.

demonstrated that rEPN firing was most prominent beginning $20 \mathrm{~min}$ after cocaine infusions, when cocaine's motivational effects have switched from rewarding to aversive. Notably, a recent study showed that optogenetically identified LHb-projecting rEPN neurons displayed monotonic encoding of valence and preferentially encode negative valence (Stephenson-Jones et al., 2016). Given that we found a large overlap between valence-encoding and cocaine 
aversion-activated rEPN neurons, it is very likely that the majority of cocaine aversion-activated rEPN neurons are LHb projecting.

A previous study had shown that the rEPN-LHb pathway encodes value and regulates dopamine firing (Bromberg-Martin et al., 2010). In the present study, we observed that rEPN responses during cocaine's "rewarding" phase correlate positively with responses to reward-predictive cues, while responses during its "aversive" phase positively correlated with responses to shock-predictive cues and shocks. This biphasic response to cocaine is similar to the responses we observed previously in LHb and RMTg neurons (Jhou et al., 2013; Li et al., 2019a), and likely contributes to those responses, as rEPN lesions attenuated cocaine-induced cFos activation in both regions. Additionally, a previous rabies tracing study indicates that the LHb neurons receiving rEPN input project to the RMTg but not the VTA (Li et al., 2019b), suggesting a critical role of serial connections between the rEPN, LHb, and RMTg in encoding aversive effects of cocaine.

However, because our behavioral tasks (cocaine CPA and runway) require learning of a cocaine avoidance response, we can only claim that the rEPN contributes to learned (conditioned) aversive associations to cocaine. We cannot determine whether it also drives unconditioned aversive effects of cocaine.

Our findings also suggest possible substrates of the so-called "opponent process theory," which posits that removal of a strong affective stimulus can produce an affective state in the opposite direction of the initial stimulus (Solomon and Corbit, 1973). Consistent with this opponency, cocaine's aversive effects occur at a time delay when blood plasma levels of cocaine are $80-90 \%$ below their initial peaks (Booze et al., 1997; Ma et al., 1999), consistent with them being because of removal of the drug rather than its presence per se. This theory has been largely used to explain drug vulnerability and relapse (Koob and Le Moal, $2008 \mathrm{~b}$ ), as the acute crash produced by abused drugs can serve as an intrinsic motivator and facilitate drug intake in self-administration and reinstatement models (Ahmed and Koob, 1998; Shaham et al., 2003; Kitamura et al., 2006; Bentzley et al., 2014). However, it should be noted that we did not test this role of drug aversion in driving intake and reinstatement, but instead tested a different role of drug aversion in preventing drug intake. Unlike the "opponent process" role in driving drug intake, which is generally observed in experienced and/ or drug-dependent individuals, this inhibitory role manifests much earlier, after only a few exposures to the drug, and many not necessarily use the same brain circuits as the later aversion-induced drug-seeking behaviors.

Notably, other studies have also implicated the rEPN-LHb pathway in cocaine's aversive effects, specifically the anxiogentic symptoms of cocaine withdrawal (Meye et al., 2016; Tan et al., 2019). Our findings corroborate those, while showing a somewhat different influence on conditioned responses to the drug itself, as rEPN inactivation in a runway operant task enhanced cocaine-seeking behaviors, while inhibiting the rEPN-LHb pathway blocked cocaine-induced CPA. Because we infused CNO 10 min after cocaine was given, when cocaine's rewarding effects are largely dissipated, and cocaine levels in blood are far below initial peaks, we posit that inhibition of the rEPN to LHb pathway specifically suppressed aversive components of cocaine rather than enhancing its rewarding components. However, we cannot rule out other interpretations, such as the possibility that removal of the aversive effects unmasked a latent residual cocaine reward, or that it could have produced rewarding effects independently of cocaine, as in previous studies in which photoinhibition of this pathway affected value-based decisionmaking (Stephenson-Jones et al., 2016).

We also found evidence that the rEPN does not drive all aversive responses to cocaine. For example, on the runway task, when muscimol infusions were replaced by saline, latency increases developed much faster than in control animals, suggesting some residual aversive conditioning had likely occurred in the earlier muscimol sessions. In particular, high latencies were observed on the very first saline trial, although all previous trials had been with cocaine. Notably, while rEPN lesions reduced cocaine-induced $\mathrm{LHb}$ cFos 2 -fold, the reduction in RMTg cFos was much less, only about one third, suggesting that between half and two-thirds of cocaine-induced cFos in the LHb and RMTg does not depend on the rEPN.

Together, our results indicate an important, though not exclusive, role of the rEPN-LHb-RMTg pathway in encoding cocaine avoidance and provide a novel mechanism that can be targeted for preventing addiction. Because the LHb and RMTg are also implicated in responses to other drugs of abuse, including alcohol, morphine, nicotine, and cannabinoids (Jhou et al., 2009, 2013; Lecca et al., 2011, 2012; Kaufling and Aston-Jones, 2015; Glover et al., 2016; Wang et al., 2017), present results suggest the possibility that the rEPN could contribute to motivational responses to those drugs as well. Future studies should focus on dissecting receptor-level mechanisms involved in this pathway, to inform the identification of specific targets and development of novel therapeutic agents.

\section{References}

Ahmed SH, Koob GF (1998) Transition from moderate to excessive drug intake: change in hedonic set point. Science 282:298-300.

Bentzley BS, Jhou TC, Aston-Jones G (2014) Economic demand predicts addiction-like behavior and therapeutic efficacy of oxytocin in the rat. Proc Natl Acad Sci USA 111:11822-11827.

Booze RM, Lehner AF, Wallace DR, Welch MA, Mactutus CF (1997) Doseresponse cocaine pharmacokinetics and metabolite profile following intravenous administration and arterial sampling in unanesthetized, freely moving male rats. Neurotoxicol Teratol 19:7-15.

Bromberg-Martin ES, Matsumoto M, Hong S, Hikosaka O (2010) A pallidushabenula-dopamine pathway signals inferred stimulus values. J Neurophysiol 104:1068-1076.

Ettenberg A (2004) Opponent process properties of self-administered cocaine. Neurosci Biobehav Rev 27:721-728.

Ettenberg A (2009) The runway model of drug self-administration. Pharmacol Biochem Behav 91:271-277.

Ettenberg A, Raven MA, Danluck DA, Necessary BD (1999) Evidence for opponent-process actions of intravenous cocaine. Pharmacol Biochem Behav 64:507-512.

Glover EJ, McDougle MJ, Siegel GS, Jhou TC, Chandler LJ (2016) Role for the rostromedial tegmental nucleus in signaling the aversive properties of alcohol. Alcohol Clin Exp Res 40:1651-1661.

Good CH, Wang H, Chen YH, Mejias-Aponte CA, Hoffman AF, Lupica CR (2013) Dopamine D4 receptor excitation of lateral habenula neurons via multiple cellular mechanisms. J Neurosci 33:16853-16864.

Jhou TC, Fields HL, Baxter MG, Saper CB, Holland PC (2009) The rostromedial tegmental nucleus (RMTg), a GABAergic afferent to midbrain dopamine neurons, encodes aversive stimuli and inhibits motor responses. Neuron 61:786-800.

Jhou TC, Good CH, Rowley CS, Xu SP, Wang H, Burnham NW, Hoffman AF, Lupica CR, Ikemoto S (2013) Cocaine drives aversive conditioning via delayed activation of dopamine-responsive habenular and midbrain pathways. J Neurosci 33:7501-7512.

Kaufling J, Aston-Jones G (2015) Persistent adaptations in afferents to ventral tegmental dopamine neurons after opiate withdrawal. J Neurosci 35:10290-10303.

Kaufling J, Veinante P, Pawlowski SA, Freund-Mercier MJ, Barrot M (2009) Afferents to the GABAergic tail of the ventral tegmental area in the rat. J Comp Neurol 513:597-621. 
Kitamura O, Wee S, Specio SE, Koob GF, Pulvirenti L (2006) Escalation of methamphetamine self-administration in rats: a dose-effect function. Psychopharmacology (Berl) 186:48-53.

Knackstedt LA, Samimi MM, Ettenberg A (2002) Evidence for opponentprocess actions of intravenous cocaine and cocaethylene. Pharmacol Biochem Behav 72:931-936.

Koob GF, Le Moal M (2008a) Addiction and the brain antireward system. Annu Rev Psychol 59:29-53.

Koob GF, Le Moal M (2008b) Review. Neurobiological mechanisms for opponent motivational processes in addiction. Philos Trans R Soc Lond B Biol Sci 363:3113-3123.

Koob GF, Stinus L, Le Moal M, Bloom FE (1989) Opponent process theory of motivation: neurobiological evidence from studies of opiate dependence. Neurosci Biobehav Rev 13:135-140.

Lecca S, Melis M, Luchicchi A, Ennas MG, Castelli MP, Muntoni AL, Pistis M (2011) Effects of drugs of abuse on putative rostromedial tegmental neurons, inhibitory afferents to midbrain dopamine cells. Neuropsychopharmacology 36:589-602.

Lecca S, Melis M, Luchicchi A, Muntoni AL, Pistis M (2012) Inhibitory inputs from rostromedial tegmental neurons regulate spontaneous activity of midbrain dopamine cells and their responses to drugs of abuse. Neuropsychopharmacology 37:1164-1176.

Levi LA, Inbar K, Nachshon N, Bernat N, Gatterer A, Inbar D, Kupchik YM (2020) Projection-specific potentiation of ventral pallidal glutamatergic outputs after abstinence from cocaine. J Neurosci 40:1276-1285.

Li, B, Piriz J, Mirrione M, Chung C, Proulx CD, Schulz D, Henn F, Malinow R (2011) Synaptic potentiation onto habenula neurons in the learned helplessness model of depression. Nature 470:535-539.

Li H, Pullmann D, Cho JY, Eid M, Jhou TC (2019a) Generality and opponency of rostromedial tegmental (RMTg) roles in valence processing. Elife 8.

Li H, Pullmann D, Jhou TC (2019b) Valence-encoding in the lateral habenula arises from the entopeduncular region. Elife 8. e41223.

Li H, Vento PJ, Parrilla-Carrero J, Pullmann D, Chao YS, Eid M, Jhou TC (2019c) Three rostromedial tegmental afferents drive triply dissociable aspects of punishment learning and aversive valence encoding. Neuron 104:987-999.e4.

Ma F, Falk JL, Lau CE (1999) Within-subject variability in cocaine pharmacokinetics and pharmacodynamics after intraperitoneal compared with intravenous cocaine administration. Exp Clin Psychopharmacol 7:3-12.

Meye FJ, Soiza-Reilly M, Smit T, Diana MA, Schwarz MK, Mameli M (2016) Shifted pallidal co-release of GABA and glutamate in habenula drives cocaine withdrawal and relapse. Nat Neurosci 19:1019-1024.

Paxinos G, Watson C (1998) The rat brain in stereotaxic coordinates, Fourth Edition. London: Academic Press.

Rajakumar N, Elisevich K, Flumerfelt BA (1994) Parvalbumin-containing GABAergic neurons in the basal ganglia output system of the rat. J Comp Neurol 350:324-336.

Shabel SJ, Proulx CD, Trias A, Murphy RT, Malinow R (2012) Input to the lateral habenula from the basal ganglia is excitatory, aversive, and suppressed by serotonin. Neuron 74:475-481.

Shaham Y, Shalev U, Lu L, de Wit H, Stewart J (2003) The reinstatement model of drug relapse: history, methodology and major findings. Psychopharmacology (Berl) 168:3-20.

Sheth C, Furlong TM, Keefe KA, Taha SA (2017) The lateral hypothalamus to lateral habenula projection, but not the ventral pallidum to lateral habenula projection, regulates voluntary ethanol consumption. Behav Brain Res 328:195-208.

Solomon RL, Corbit JD (1973) An opponent-process theory of motivation. II. Cigarette addiction. J Abnorm Psychol 81:158-171.

Stephenson-Jones M, Yu K, Ahrens S, Tucciarone JM, van Huijstee AN, Mejia LA, Penzo MA, Tai LH, Wilbrecht L, Li B (2016) A basal ganglia circuit for evaluating action outcomes. Nature 539:289-293.

Tan D, Nuno-Perez A, Mameli M, Meye FJ (2019) Cocaine withdrawal reduces $\mathrm{GABAB} R$ transmission at entopeduncular nucleus - lateral habenula synapses. Eur J Neurosci 50:2124-2133.

Wang J, Li M, Wang P, Zha Y, He Z, Li Z (2017) Inhibition of the lateral habenular CaMK abolishes naloxone-precipitated conditioned place aversion in morphine-dependent mice. Neurosci Lett 653:64-70. 